\title{
Cómo contratar un servicio de posicionamiento web
}

\author{
Por Cristòfol Rovira, Lluís Codina y Mari-Carmen Marcos
}

Resumen: Se presenta un conjunto de recomendaciones para empresas o profesionales que necesiten contratar un servicio de posicionamiento en buscadores (SEO) con el fin de externalizar esta tarea. Las recomendaciones se articulan en torno a cuatro fases: (1) obtención inicial de información sobre proveedores de servicios SEO, (2) contrastación de la información, (3) contratación y (4) seguimiento. Se presentan también las principales características del llamado black hat SEO, es decir, una clase de posicionamiento perseguido por los motores de búsqueda por utilizar malas prácticas.

Palabras clave: Contratación de servicios, Posicionamiento en buscadores, SEO, Malas prácticas, Black hat SEO.

Title: How to hire a web positioning service

Abstract: A set of recommen-

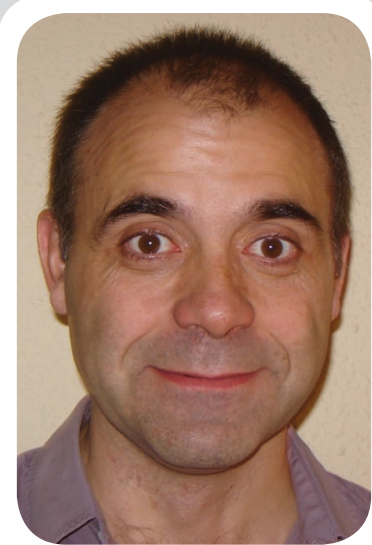

Cristòfol Rovira es profesor de la Universitat Pompeu Fabra (UPF) en el área de biblioteconomía y documentación. Imparte docencia en las titulaciones de publicidad y relaciones públicas y traducción e interpretación. Es coordinador del máster interuniversitario UB/UPF sobre gestión de contenidos digitales y director del máster online en buscadores, del máster online en documentación digital e investigador del Grupo DigiDoc del departamento de periodismo y de comunicación audiovisual de la UPF.

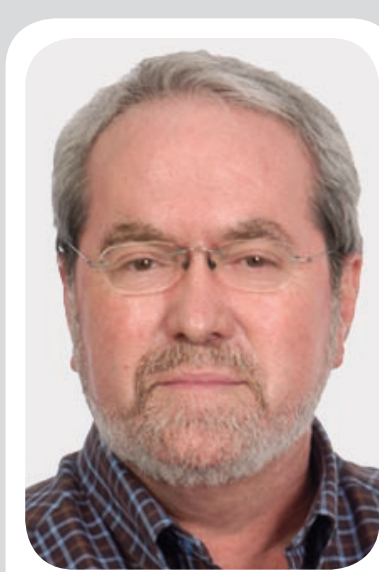

Lluís Codina es profesor titular de la Universitat Pompeu Fabra y coordinador del área de biblioteconomía y documentación de la citada universidad. Imparte docencia en las titulaciones de periodismo y de comunicación audiovisual. Es coordinador del Grupo de Investigación DigiDoc de la misma universidad y codirector del máster online en buscadores y del máster online en documentación digital. Participa en el máster Interuniversitario UB/UPF sobre gestión de contenidos digitales.

dations are presented that are

intended to be useful for the externalization of a SEO service. The recommendations are articulated around four phases: (1) initial information acquisition, (2) information contrast,

(3) hiring, (4) monitoring. The main characteristics of black hat SEO practices, or bad practices in SEO, are presented.

Keywords: Service hiring, Search engine optimization, Bad practices, Black hat SEO.

Rovira, Cristòfol; Codina, Lluís; Marcos, Mari-Carmen. “Cómo contratar un servicio de posicionamiento web”. El profesional de la información, 2009, marzo-abril, v. 18, n. 2, pp. 229-236.

DOI: 10.3145/epi.2009.mar.14

\section{Introducción}

NUESTRA INTENCIÓN ES PROPORCIONAR RECOMENDACIONES sobre cómo contratar un servicio SEO (search engine optimization o search engine positioning), es decir, de posicionamiento en buscadores, en el caso de que decidamos externalizar esta tarea.

Posicionar un sitio en relación con los buscadores consiste principalmente en que el sitio ocupe buenas posiciones, las primeras, en las páginas de resultados de los motores de búsqueda. Las acciones de análisis y las medidas que se adoptan en el campo del SEO van siempre destinadas a que, dada una palabra clave (o una serie de ellas), las páginas del sitio aparezcan en buenas posiciones cuando los internautas realizan búsquedas usando tales palabras clave.

Dado que la página de resultados de un buscador presenta 10 re- ferencias, por "buenas posiciones" entendemos encontrarse, a ser posible, entre estas primeras diez refe-

\section{"El objetivo es que nuestro sitio web se encuentre entre las 10 primeras referencias en la página de resultados de un buscador"}


rencias $y$, en el peor de los casos al menos entre las 20 primeras.

Estas recomendaciones también pueden ser útiles para las empresas o profesionales que suministren este tipo de servicios ya que les permitirán evitar malas prácticas o errores que puedan generar desconfianza en sus potenciales clientes. No obstante, el texto está escrito siempre desde la perspectiva de un profesional de la documentación o en general, del responsable de un sitio web que necesita contratar un servicio SEO.

La metodología para este trabajo ha sido la siguiente: en primer lugar, los autores han contrastado su experiencia en campañas de posicionamiento en las que han obtenido resultados positivos con las ofertas de servicios que hacen las empresas de SEO; en segundo lugar han entrevistado a expertos de reconocido prestigio en la materia $^{1}$; en tercer lugar, han analizado fuentes y bibliografía sobre posicionamiento web que incluyen información sobre el llamado black hat SEO, es decir, sobre las malas prácticas en posicionamiento web; en cuarto lugar se han realizado búsquedas sobre posicionamiento web y se han analizado las ofertas de las empresas que proporcionan servicios SEO. Por último, las recomendaciones de este trabajo se han beneficiado del hecho de haber sido sometido a debate en diversas sesiones de un seminario de investigación sobre publicación digital y buscadores en general en el que participan expertos de varias universidades $^{2}$.

\section{Riesgos y detalles}

Aunque probablemente la mayor parte de las empresas SEO siguen un buen código deontológico, el primer paso cuando contratamos una de ellas consistirá en asegurarnos de su solvencia y profesionalidad. No es solamente una razón económica (no malgastar dinero), sino que una mala práctica podría implicar la desaparición de nuestra web del índice de los buscadores, con el consiguiente desprestigio y descalabro económico que ello implicaría, mucho más allá de las tarifas (relativamente reducidas) malgastadas de esta clase de servicios.

¿Qué queremos decir con "malas prácticas"? Nos referimos a que al conocer con cierto detalle los criterios que los buscadores tienen en cuenta para ordenar los resultados en su ranking, algunas empresas de SEO intentan "trucar" los resultados con la intención de conseguir mejores posiciones para sus clientes de una forma rápida y fácil, a ser posible sin invertir ni un céntimo en crear contenidos para el sitio ni hacérselo invertir al cliente y así parecer muy eficientes delante del mismo.

\section{"Las malas prácticas son detectadas fácilmente por los buscadores y los sitios web que las aplican son sancionados"}

La contradicción básica es que los buscadores quieren posicionar bien únicamente las páginas con contenidos de calidad para la palabra clave de búsqueda. Pero crear tales contenidos no es ni rápido ni gratis. A partir de aquí, las malas prácticas intentan que un sitio sin contenido de calidad quede situado en buenas posiciones en las páginas de resultados del buscador. Ahora bien, por razones de supervivencia, los buscadores intentarán "castigar" esas prácticas ya que, de generalizarse, les harían perder la confianza de los usuarios, y de paso el suculento mercado de miles de millones de dólares en publicidad que manejan ("resultados patrocinados") gracias a la amplia audiencia de la que pueden presumir delante de los anunciantes.

Por supuesto, algunas empresas de SEO pueden responder que los buscadores son también empresas y que cada uno tiene sus intereses, por lo que en este terreno todo es lícito. No nos dejemos llevar por estos argumentos porque:

- Las malas prácticas son fáciles de detectar por los buscadores, y las webs que las aplican son sancionadas con unas posiciones muy bajas o con la expulsión del índice del buscador

- La ética en este caso va más allá del respeto a las normas de los buscadores; si un sitio web está en un listado de resultados sin tener nada que ver con lo que buscaba el usuario o sin tener ningún contenido del más mínimo interés se está produciendo una falta de ética.

- En realidad, a medio o a largo plazo, a nadie le interesan las malas prácticas: al buscador por las razones alegadas; al cliente que contrata el servicio porque el tráfico adquirido será "pan para hoy y hambre para mañana", ya que no logrará conversiones $^{3}$ y puede ver su sitio expulsado del índice del buscador. La única beneficiada será la empresa SEO, aunque seguramente deberá ir cambiando de nombre y de dominio para no verse expulsada del mercado.

Entre las prácticas no éticas más comunes se encuentran las siguientes:

\section{Enlaces de llegada (in-links)}

- Redes de dominios o granjas de enlaces desde donde unos sitios enlazan a otros y todos se enlazan entre sí, sin tener relación temática ente ellos.

- Compra de enlaces (muchas veces para entrar en granjas de enlaces).

- Comentarios en foros y blogs de forma automática incluyendo 
un link (enlace) al sitio web que se quiere posicionar.

- Link-bombing, poniendo en una gran cantidad de páginas un link al sitio web con un anchor (ancla) que no se corresponde con los contenidos de los que trata.

Un incremento excesivo o estadísticamente atípico del número de enlaces de entrada que apuntan a una web en un breve espacio de tiempo puede ser considerado por lo buscadores un indicador de malas prácticas (spam de enlaces).

\section{Textos de las páginas}

- Ocultar texto escribiendo con un color de fuente igual al color de fondo, de forma que las personas no lo veremos pero los robots sí lo indexan.

- Usar un tamaño de letra muy pequeño que las personas apenas puedan leer, pero que los robots indexan.

- Abusar de la repetición de las palabras clave para las que se quiere posicionar la página repitiéndolas de forma poco natural con una alta densidad, es decir, repitiendo muchas veces la misma palabra en el cuerpo de una página con el fin de hacer "creer" al buscador que la página es muy relevante en relación con esa palabra al presentar un ratio de la palabra clave en cuestión muy alto en relación con el total de palabras de la página.

\section{Versiones de los sitios web}

- Técnicas de cloaking, que consisten en proporcionar a los robots un texto distinto del que pueden ver las personas. La forma de hacerlo es mediante unas páginas (denominadas doorways) que redireccionan a otras según el servidor detecte que la petición de la página la realiza un robot o un internauta.

\section{URL del sitio}

- Registrar dominios que inducen a engaño: por ejemplo, $w w w$. gogle.com (o sea, Google con una sola “o”) para atraer el tráfico de los que teclean mal o conocen mal el nombre de alguna empresa o marca muy famosa.

- Crear URLs con nombres de directorios y/o de archivos de marcas comerciales muy buscadas, por ejemplo $w w w$.verybadsite. com/sony, aunque la empresa del sitio (verybadsite) no tenga ninguna relación legal ni contractual con la empresa cuyo nombre forma parte de la URL (Sony), ni aporte información sobre ella. Solamente se pretende atraer tráfico usando la conocida debilidad de Google por los textos de las URLs a la hora de calcular el ranking de su página de resultados.

- Una variante puede consistir en registrar subdominios que incluyen nombres de marcas o productos sin tener relación con la empresa propietaria y con los mismos fines anteriores, por ejemplo: sony. verybadsite.com.

\begin{tabular}{|c|}
\hline $\begin{array}{c}\text { Principales prácticas } \\
\text { de black hat }\end{array}$ \\
que los buscadores pueden \\
detectar
\end{tabular}

Si tenemos en cuenta la denominada "contradicción esencial", que se puede resumir en que todos queremos que nuestro sitio quede bien posicionado pero nadie quiere que los motores le pongan basura en la página de resultados cuando usa Google o Yahoo!, llegaremos a la conclusión que la famosa expresión "el contenido es el rey" cobra toda su dimensión en materia de posicionamiento.
Los ingenieros de los buscadores buscan con ahínco el desarrollo de algoritmos de ranking que beneficien a los sitios con buenos $\mathrm{y}$ abundantes contenidos, y sitios que los renuevan o añaden con frecuencia. A poco que lo pensemos veremos que es lógico que sea así. De otro modo, los buscadores dejarían de tener usuarios. ¿Nos imaginamos lo contrario: buscadores que promocionen los sitios con peores materiales? Afortunadamente, todo su empeño (aunque no siempre aciertan) lo ponen en evitar sitios con información de interés ridículo.

Esto no significa, por desgracia, que sea suficiente tener un buen contenido, ya que hay diversas barreras técnicas, así como errores conceptuales en los que caen los sitios, que impiden que ese buen contenido (si lo hay) sea adecuadamente representado en la página de resultados del buscador, y es aquí donde entran los buenos profesionales del SEO.

Por mucho que insistan, debe saberse que no hay recetas milagrosas para asegurar el éxito en este apartado, aunque algunas recomendaciones sin duda reducirán considerablemente el riesgo. Una buena intuición también juega un papel a veces fundamental, ya que en este contexto los pequeños detalles pueden ser determinantes.

Teniendo en cuenta lo anterior, las recomendaciones que a continuación presentamos están organizadas temporalmente en cuatro momentos del proceso de contratación y uso de un servicio de SEO:

- Obtención inicial de información sobre proveedores de SEO

- Contrastación de la información sobre el servicio

- Contratación del servicio

- Seguimiento.

En cada uno de estos momentos se puede realizar una serie de comprobaciones que lleven a aumen- 


\section{"Hay que obtener toda la información posible de las empresas que proporcionan servicios de SEO"}

tar o disminuir la confianza en las compañías seleccionadas, que ayuden a tomar la decisión de compra y que finalmente permitan hacer un seguimiento efectivo del servicio contratado.

\section{Obtención inicial de información sobre proveedores de SEO}

No resultará difícil obtener información de empresas que proporcionan servicios de SEO. Una simple búsqueda en Google o Yahoo! nos dará un primer listado. Naturalmente el propio posicionamiento de este tipo de empresas en la sección de resultados orgánicos es un indicador de su habilidad; por tanto no podemos menospreciar la posición en la que quedan situadas las empresas del sector en estas búsquedas. Algunas de las frases más habituales utilizadas para encontrar empresas en este sector son:

- posicionamiento buscadores

- posicionamiento Google

- servicios de posicionamiento

- "posicionamiento en buscadores"

- "posicionamiento web"

- "posicionamiento en Google"

- "servicios de posicionamiento"

También se deben revisar los enlaces patrocinados que incluyen los buscadores. A menudo las empresas más activas son las que se encuentran en las primeras posiciones en este apartado, pero en cualquier caso no se deje impresionar, al fin y al cabo, los enlaces patrocinados dependen del dinero que se ha pagado por la posición del anuncio y no tiene porque estar relacionada la disponibilidad de fondos con un buen servicio.

Normalmente resultará más fácil y directo el trato con empresas del propio país. También puede resultar más sencilla la obtención de garantías o referencias de empresas cercanas. No obstante, con un mundo globalizado, la cercanía o lejanía dejan de ser geográficas. No hay barreras, ni para lo malo ni para lo bueno. En todo caso, necesitaremos garantías de que la empresa (sea o no de nuestro país) cuenta con personal capaz especializado en el idioma de nuestra web.

El idioma de trabajo de la empresa puede ser un elemento a tener en cuenta, puesto que no podemos renunciar a un contacto telefónico fluido. Por otro lado, la contratación puede resultar más económica si la empresa reside en un país donde el euro sea una moneda fuerte. No obstante, este factor no debería ser tenido en cuenta hasta las fases finales del proceso de contratación.

Sería interesante pedir también que las empresas SEO especifiquen en su oferta las principales técnicas a utilizar, o al menos los factores sobre los que van a trabajar. La regla o consejo subyacente aquí sería que cuantos más factores estén dispuestas a considerar, más completo será el servicio, y si indican sus técnicas, después se las podrá valorar sobre las mismas.

Resultará también útil visitar algún directorio para ver cuántas empresas SEO contiene. Una empresa SEO que se precie no debería descuidar su inclusión en los principales directorios generalistas como Dmoz o Yahoo! Directorio.

Por supuesto, resultaría mucho más interesante un directorio especializado en empresas de SEO, pero hasta donde nosotros hemos podido comprobar, actualmente no hay ninguno con garantías de inde- pendencia. Hay que ser muy prudente al buscar directorios de empresas SEO en un buscador puesto que los resultados nos llevan en realidad a directorios creados para proporcionar enlaces de entrada de manera "no natural", es decir, directorios proveedores de enlaces creados por empresas de posicionamiento para sus clientes o bien a falsos directorios cuyo principal finalidad es exhibir anuncios procedentes del programa AdSense de Google.

Además, los directorios para proporcionar enlaces de entrada (llamados "Directorios SEO") a veces están al borde de lo que algunos buscadores pueden considerar no ético. En todo caso, cabe constatar que es una actividad en auge.

Otra buena estrategia para localizar un buen servicio de SEO es localizando antes un experto que muy probablemente será la cabeza visible de una empresa de este tipo. Aunque se ha publicado alguna lista de expertos SEO de nuestro país, la mejor manera de encontrar estos especialistas es por medio de sus blogs. Se puede usar la búsqueda avanzada de Google Blogs, con los términos "SEO" o "posicionamiento" en el título del blog o buscar en Technorati los mismos términos en tags de post en español de máxima autoridad. Los rankings de blogs también pueden ser un instrumento útil en este contexto, siempre y cuando se puedan aplicar filtros de lengua, país o materia y que exista la categoría "SEO" o "posicionamiento en buscadores".

http://www.posicionalia.com/ 20070112-listado-de-expertos-seo. html

http://www.bloguzz.com/index/ rank/cat/seo/lang/spanish

Por otra parte, se encuentra en España la filial de Sempo (Search Engine Marketing Professional Organization), una asociación profesional internacional de empresas 


\begin{tabular}{|c|c|c|c|}
\hline \multicolumn{4}{|c|}{$\begin{array}{l}\text { Servicios de Red }>\text { Promoción y publicidad } \\
\text { Directorio }>\text { Zonas geográficas }>\text { Paises }>\text { España }>\text { Economi } \\
\text { publicidad }\end{array}$} \\
\hline Buscar & todos los sitios $\bigcirc_{\text {sólo sitios de España }}$ sólo esta categoría & Buscar & $\frac{\text { búsqueda avanzada }}{\underline{\text { Sugiera un sitio }}}$ \\
\hline
\end{tabular}

SITIOS ENCONTRADOS EN ESTA CATEGORIA

Listado alfabético | Por popularidad (Quéesesto?)

- Activ, S.L. E⿱ E- Brindan servicios de presencia corporativa en la Internet y otros servicios relacionados a la Internet.

- Afroweb 昌. Dedicado a promocionar sitios.

- Alta-en-Buscadores.com 宣- Servicio anual de alta y posicionamiento de sitios web en los principales buscadores. Informe de consultoria previo y panel de control online.

- Altaenbuscadores.com@

- Altaweb 昼- Servicios de márketing. promoción y alta en buscadores.

- Atraczion 是- Servicio de registro en buscadores, promoción y posicionamiento de sitios Web.

- Beatriz Martinez, S.L. E⿱ E⿱ - Entidad que provee varios servicios relacionados con la Red incluyendo el registro de dominios, promociones en línea y una feria de comercio virtual.

- Canary Companies $\underline{\text { E⿱ }}$ - Registro de dominios, alojamiento, diseño de páginas web, directorio y enlaces.

- Emred 宣- Diseño de páginas web y aplicaciones de comercio electrónico B2B y B2C con tecnología Java. Flash y Html dinámico.

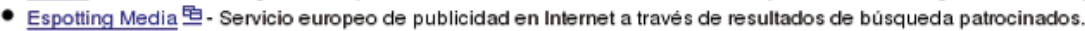

- Foro Digital S.L. 宣- Proyectos multimedia, consultoria informatica y de Internet, asi como márketing y publicidad en la Red.

Apartado de Yahoo.es sobre ...Productos y servicios para empresas (B2B) > Servicios de Internet > Servicios de Red > Promoción y publicidad. http://es.dir.yahoo.com/...

de marketing y posicionamiento en buscadores. Aunque no están todos los que son (ni mucho menos), no estaría de más revisar el listado de empresas asociadas, especialmente porque últimamente han propuesto un proceso de certificación para agencias de servicios SEO y SEM (search engine marketing).

Hay otros sitios donde buscar empresas de SEO pero son menos eficaces que un buscador clásico como Google o Yahoo!. Se pueden usar buscadores de noticias o incluso YouTube. A menudo el canal más eficaz es el "boca a oreja": pregunte a personas de su confianza que sepa que han utilizado esta clase de servicios.

Cabe señalar que seguramente hay que desconfiar ante empresas que envían mensajes de promoción o de propuestas de servicios mediante correo electrónico no solicitado. Son prácticas agresivas que ya nos indican el talante de la empresa. Es muy probable que también sean agresivas en sus campañas de posicionamiento hasta el punto de realizar prácticas poco éticas también en este campo (y no olvidemos que, además de los reparos éticos, que deberían ser suficientes en sí mismos, nos arriesgamos a la penalización por parte de los buscadores).

\section{Contrastar la información obtenida}

Como resultado del primer paso, es decir, después de haber buscado en los medios indicados, se supone que deberá tener 3, 4 ó 5 empresas candidatas. Sobre estos candidatos deberá recabar información adicional. Pregunte a sus colegas y consulte en internet la información disponible sobre ellas:

- Revise con detenimiento las sedes web de las empresas seleccionadas. Es conveniente confirmar que los datos de los responsables se muestran claramente y con todo detalle. Revise las características de diseño y la información que contiene. Los detalles aquí son fundamentales.

- Compruebe que los sitios web de las empresas seleccionadas están en Google y Yahoo!. Si no las ve, un posible motivo es que hayan sido sancionadas por aplicar en sus propios sitios web prácticas consideradas no éticas por los buscadores.
- Averigüe el PageRank de las empresas seleccionadas.

- Revise las páginas web que apuntan a las empresas seleccionadas. Resultará de interés saber cuántos enlaces reciben y de quién proceden.

- Revise el código fuente de los sitios web de estas empresas para comprobar buenas prácticas: uso de metadatos, hoja de estilos, etc.

- Observe si tienen algún sello de validación del código sobre la sintaxis o la accesibilidad. Es un detalle que denota interés por las cosas bien hechas.

- Busque el nombre de las empresas en blogs. A menudo se trata de blogs creados por la propia empresa para promocionarse de forma explícita. Revise el estilo y el tipo de mensajes de estos blogs. También podría tratarse de un blog sobre SEO, sin vinculación con la empresa y con comentarios de interés.

- Busque el nombre de las empresas en buscadores de noticias, podría obtener algún dato adicional.

- Revise en Wayback Machine (accesible desde Archive.org) desde 
qué año se encuentra disponible la web de las empresas seleccionadas y qué diseños ha tenido anteriormente. Podrá detectar desde qué año se dedica al posicionamiento, si ha habido cambios de orientación en el negocio, etc.

- Visite a los clientes de las empresas seleccionadas (si se lo puede permitir). Las mejores empresas informan siempre sobre sus casos de éxito. Será un indicador positivo si el propio sitio web de la empresa SEO presenta esta información. Visite las webs de los clientes para comprobar su PageRank; observe también el código para detectar posibles prácticas fraudulentas, como texto o vínculos ocultos. A continuación visite las webs que apuntan a estos clientes para identificar posibles granjas de enlaces.

\section{Contratar}

Como resultado del paso anterior deberá tener ahora sus 3, 4 ó 5 empresas iniciales marcadas con una primera ordenación en función de su preferencia (después de revisar la información obtenida). El siguiente paso consistirá en entrar en detalle sobre las condiciones de contratación que ofrecen estas compañías.

En este sentido es útil recordar que el propio Google informa de manera muy clara de que "nadie puede garantizarle el puesto número 1 en el ranking de Google". Por tanto, desconfíe de las empresas que le den esta garantía, incluso cuando se hable de estar entre los 10 primeros resultados. A menudo este tipo de garantías se refieren al uso de palabras clave poco comunes o frases muy largas que los clientes raramente utilizarían y sobre las cuales no hay competencia. También se suele usar el nombre de la empresa para demostrar la obtención de primeros resultados, cuando en la mayoría de las ocasiones los nombres propios no necesitan una campaña de SEO para salir bien posicionados, puesto que no tienen competencia. En otras ocasiones las primeras posiciones se obtienen por pago por clic, intentando confundir al usuario poco experto que no diferencia entre los enlaces orgánicos y los patrocinados.

Otras veces la garantía se basa en las posiciones relativas obtenidas con otros clientes. Hay que ser precavido ante este tipo de afirmaciones. Primero hay que comprobarlo de forma clara evitando la confusión de enlaces patrocinados con resultados orgánicos, y segundo hay que analizar el tipo de empresa, las palabras clave usadas, su competidores, el sector, etc., para determinar hasta qué punto nuestra empresa es equiparable y por tanto si resulta lógico pensar que lo que han hecho con ellos también lo podrán hacer con nosotros.

Algunas empresas de SEO garantizan buenas posiciones proponiendo de forma abierta el uso de prácticas fraudulentas. Aparte de no cumplir con las consideraciones éticas impuestas por las reglas de los propios buscadores, y de esta forma quedar expuestos a las penalizaciones que aplican, no es una práctica efectiva puesto que más que enganar a los buscadores lo que se consigue es engañar a los usuarios que llegan a la página por error. Por tanto deberá desconfiar, y mucho, ante este tipo de propuestas.

Las empresas de SEO no pueden garantizar resultados concretos puesto que no controlan los posibles cambios que los buscadores puedan realizar en el futuro. Cualquier campaña de SEO usará la información relativa a la historia de los buscadores hasta la fecha pero ¿quién puede asegurar como será el posicionamiento en el futuro? Si difícilmente se conoce el funcionamiento actual de los buscadores, ¿cómo se pueden dar garantías del funcionamiento futuro? Por ejemplo, Google está retocando constantemente sus algoritmos de ordenación para luchar precisamente contra las prácticas fraudulentas y por ello, lo que sirve para hoy es posible que para mañana no sirva.

Por otro lado, tampoco pueden trabajar la optimización para todas las variantes de búsquedas que se efectuarán, ni tampoco es posible predecir todas las consultas que los interesados en determinados campos o servicios van a introducir en los buscadores.

Igualmente, hay otros factores que no se podrán cambiar en una campaña de SEO, por ejemplo, la historia del sitio web, ¿cuánto tiempo lleva en internet?, ¿con qué contenidos? El pasado de una web influye en su posicionamiento y no se puede cambiar de un día para otro.

En igualdad de condiciones es muy probable que salga antes una página con las palabras clave de la búsqueda como parte de su URL que otra página sin este elemento. Conocido este factor, hay algunas empresas de SEO que facilitan la capacidad para recoger palabras clave en las URL aunque sus páginas no las tengan. Evalúe con mucha atención este tipo de ofertas que implicarán la instalación de un software específico y una dependencia futura del mismo.

En definitiva, el apartado de garantías de un contrato de SEO deberá mirarse con lupa y, en general, serán más fiables las garantías que den en relación con unos servicios concretos que se realizarán en unos plazos determinados que cualquier otro tipo de garantías relacionadas con la aparición en el índice de la sede web en los buscadores. Es posible que estas garantías de posición sean tan ambiguas que bordeen el fraude. En particular, entendemos que la garantía relacionada con los servicios debería reunir, como mínimo, los siguientes elementos:

- Asesoramiento sobre los contenidos. El posicionamiento depende en gran medida de los con- 
tenidos, por tanto la compañía de SEO debería dar consejos oportunos sobre cómo modificar, editar o ampliar los contenidos de la web en el marco de una campaña de posicionamiento y con relación a unas determinadas palabra clave.

- Asesoramiento sobre una política seria de captación de enlaces de llegada o entrada, que son, junto a los contenidos, uno de los principales factores de posicionamiento. Por tanto, es imprescindible que la empresa de SEO diseñe las líneas estratégicas para una campaña específica de captación de enlaces, obviamente sin contemplar la contratación de granjas de enlaces. Hay que ser muy prudente en este punto puesto que la obtención de enlaces es altamente susceptible a prácticas poco claras o incluso fraudulentas.

En las condiciones de contratación será muy interesante que se especifique de forma pormenorizada a qué se dedicará el dinero que pagamos, indicando tareas y horas. Desconfíe de presupuestos con grandes partidas sin conocer en detalle qué incluye cada apartado.

Finalmente, exija que en el contrato figure explícitamente que la compañía se compromete a respetar las directrices y recomendaciones de los buscadores (esto es, que se compromete a aceptar las directrices de lo que los buscadores consideran prácticas no abusivas). Esto le dará una garantía adicional en caso de que haya un contratiempo con algún buscador.

\section{Seguimiento}

La fase final consistirá en hacer un seguimiento de los servicios contratados, sobre todo de aquellos elementos que sean susceptibles de incurrir en fraude con los contenidos y los enlaces de entrada.

\section{Enlaces}

Deberá controlar que la compañía de SEO no haya colocado enlaces de entrada en su página hacia otros clientes y desde los otros clientes hacia su página. Si los contenidos están relacionados, no habría ningún conflicto en el intercambio de enlaces, el aspecto negativo está en que algunas empresas de SEO ejecutan esta práctica de forma sistemática con el propósito de aumentar el PageRank y sin que los contenidos tengan nada que ver. Además es fundamental revisar que estos vínculos no sean ocultos a ojos de un observador, pero operativos para el robot o spider de un buscador. Esto se consigue, como se ha explicado antes, dando el mismo color al texto del enlace que al fondo; los buscadores lo detectarán cuando indexen de nuevo el sitio web y podrá ser sancionado con una posición muy baja.

Tampoco será aceptable basar la política de enlaces de entrada a partir de sitios web controlados por la empresa de SEO, ya sean directorios $\mathrm{SEO}$, como los mencionados al principio, o desde otro tipo de portales creados específicamente con este fin. Cuando termine la relación con la empresa, ¿qué pasará con estos enlaces?

\section{Contenido}

Hay que evitar "páginas puerta" (doorways), en las cuales se coloque una gran cantidad de palabras clave con el fin de captar una gran cantidad de visitas provenientes de buscadores con la excusa de que estas páginas serían relevantes para un mayor número de consultas. Es completamente falso, porque una sola página no puede ser relevante para una amplia gama de palabras clave.

\section{Tráfico}

Finalmente, recuerde que realizar una campaña de optimización para buscadores tiene como primer propósito aumentar el tráfico de visitas al sitio web, y en segundo lugar (el único que realmente cuenta muchas veces) obtener conversiones, es decir, conseguir aquello que se supone esperamos que haga el público una vez visite nuestra web (descargar un archivo, suscribirse, comprar, leer un contenido, adherirse a una campaña, etc.).

Por lo tanto, paralelamente a la entrega de informes de posicionamiento por parte de la empresa SEO, haga un seguimiento del número de visitas que recibe y compárelo con las que recibía antes de iniciar la campaña. En este seguimiento, si la aplicación de análisis se lo permite (Google Analytics, por ejemplo), podrá saber cuántas visitas proceden de resultados obtenidos en buscadores.

\section{Conclusiones}

Se han dado algunas recomendaciones sobre qué tener en cuenta en el momento de contratar un servicio de SEO basadas en la experiencia de malas prácticas en un sector económico muy agresivo en el que hay que ser precavido. Las prácticas opacas no son la excepción.

A menudo la información que podemos obtener en internet permitirá confirmar las referencias obtenidas en otros contextos, ya sea en diversos medios publicitarios, por informaciones de colegas, congresos... Otras veces las referencias iniciales las podremos obtener en la propia web y buscar a partir de ellas. En todo caso, es esencial establecer un procedimiento sistemático que descarte las empresas poco serias que podrían incluso empeorar en lugar de mejorar nuestra posición en los listados de resultados de los buscadores.

\section{Agradecimientos}

Este trabajo ha sido financiado por el Ministerio de Educación y Ciencia, como parte del proyecto HUM2004-03162/FILO. 


\section{Notas}

1. Las entrevistas se han publicado en el Anuario Hipertext.net como parte del número de mayo de 2008

http://www.hipertext.net/web/pag280.htm

2. Seminario DigiDoc

www.digidocweb.net

3. Una conversión es la acción que esperamos que realicen los internautas cuando llegan a nuestra página. Puede ser descargar un fichero, realizar una suscripción, utilizar un servicio, etc Las conversiones son, de este modo, una forma de medir el éxito relativo de una campaña de posicionamiento.

\section{Fuentes}

Benítez, Magali. Un vistazo a la problemática de las garantías para posicionar páginas web en el top ranking de Google. 7 de septiembre de 2004 http://www.poliedric.com/docs/garantias_seo. php

Mora, Albert. Elegir una empresa de SEO -213 de abril de 2007.

http://www.wwwisibility.com/2007/04/13/elegiruna-empresa-de-seo-2/

Google. Centro de asistencia para webmasters. ¿Qué es un SEO? ¿Me recomienda Google que trabaje con compañías que ofrecen ajustar mi sitio a los requisitos de Google?

http://www.google.com/support/webmasters/bin/ answer.py? $h l=e s \&$ answer $=35291$

Directorio Dmoz sobre empresas de márqueting en internet en español:

http://www.dmoz.org/World/Espa\%c3\%b1ol/ Negocios/Marketing/Marketing_en_Internet/

Guía About sobre prácticas black hat. http://websearch.about.com/od/seononos/a/ spamseo.htm

Lorsh, Tomy. Ojo Buscador. En España no hay SEOs. 29 de mayo de 2006.

http://www.ojobuscador.com/2006/05/29/enespana-no-hay-seos/

Omnibius: directorio de directorios SEO con más de 900 entradas. http://www.omnibius.com/

Listado de directorios en la categoría SEO \& gratuitos.

http://www.omnibius.com/directory/GetListBy Charact/seo/free

Listado de expertos SEO. Posicionalia. Posicionamiento y optimización web en buscadores de internet.

http://www.posicionalia.com/20070112-listadode-expertos-seo.html

Sempo España.

http://www.sempo-spain.com/

Ranking de blogs SEO Español - Bloguzz. http://www.bloguzz.com/index/rank/cat/seo/ lang/spanish

Top Blogs - SEO - Red de bloggers Alianzo. http://www.alianzo.com/es/top-blogs/lang/ espanol/seo

\section{Referencias}

Ferrer, Antonia. La economía social en la sociedad de la información. "Hipertext.net", 2004, n. 2 .

http://www.hipertext.net

Cedano, Rafael. "Qué es el black hat SEO?" Seotalk.es, Marzo 2007.

http://www.seotalk.es/black-hat-seo/

Loveday, Lance; Niehaus, Sandra. Web design for ROI. Berkeley: New Riders, 2008.

Maciá, Fernando; Gosende, Javier. Posicionamiento en buscadores. Madrid: Anaya, 2007.

Marcos, Mari-Carmen et al. "Evaluación del posicionamiento web en sistemas de información terminológicos online". Hipertext.net, 2006, n. 4.

http://www.hipertext.net/web/pag267.htm

Marcos, Mari-Carmen. "Engañar a los buscadores". Anuario ThinkEPI 2008, pp. 50-53.

Pedraza-Jiménez, Rafael; Codina, Lluís; Rovira, Cristòfol. "Web semántica y ontologías en el procesamiento de la información documental". El profesional de la información, 2007, v. 16, n. 6 , pp. 569-578.
Ramos, Andreas; Cota, Stephanie. Insider SEO and PPC. Fremont: Jain Publishing, 2006

Rognerud, Jon. Ultimate guide to search engine optimization. Wisconsin: EP Media, 2008

Rovira, Cristòfol; Marcos, Mari-Carmen "Posicionamiento en buscadores de blogs de interacción persona-ordenador y usabilidad". En: Redondo Duque et al. (ed.) Interacción 2006: VII Congreso intl. de interacción persona-ordenador. Puertollano (Ciudad Real) Interacción'2006.

http://chico2.inf-cr.uclm.es/i2006/

Rovira, Cristòfol; Marcos, Mari-Carmen Codina, Lluís. "Repositorios de publicaciones digitales de libre acceso en Europa: análisis y valoración de la accesibilidad, posicionamiento web y calidad del código". El profesional de la información, v. 16, n. 1. pp. 24-38.

Sostre, Pedro; LeClaire, Jennifer. Web analytics for dummies. Hoboken: Wiley, 2007.

Thurow, Shari. Search engine visibility. Berkeley: New Riders, 2003

Walter, Aarron. Building findable websites Berkeley: New Riders, 2008.

\section{Cristòfol Rovira, Lluís Codina y Mari-Carmen Marcos}

Departamento de Comunicación, Universitat Pompeu Fabra.

Roc Boronat, 138. 08018 Barcelona.

Tel.: +34-935421311

cristofol.rovira@upf.edu

http://www.cristofolrovira.com

lluis.codina@upf.edu

http://www.lluiscodina.com

mcarmen.marcos@upf.edu

http://www.mcmarcos.com

\section{Te damos los ingredientes...}

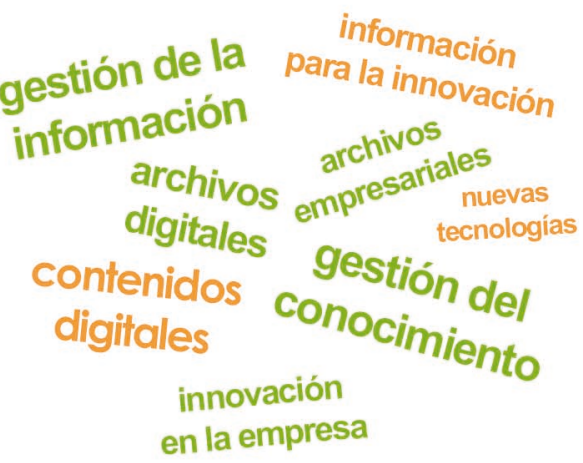

